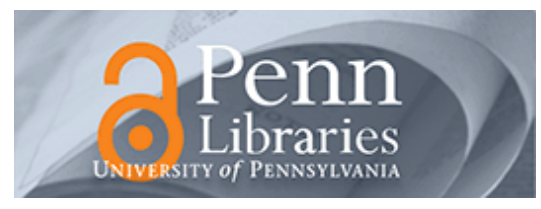

University of Pennsylvania

ScholarlyCommons

Statistics Papers

Wharton Faculty Research

1979

\title{
Hammersley's Law for the Van Der Corput Sequence: An Instance of Probability Theory for Pseudorandom Numbers
}

A del Junco

Ohio State University

J Michael Steele

University of Pennsylvania

Follow this and additional works at: https://repository.upenn.edu/statistics_papers

Part of the Physical Sciences and Mathematics Commons

\section{Recommended Citation}

Junco, A. d., \& Steele, J. M. (1979). Hammersley's Law for the Van Der Corput Sequence: An Instance of Probability Theory for Pseudorandom Numbers. The Annals of Probability, 7 (2), 267-275.

http://dx.doi.org/10.1214/aop/1176995087

At the time of publication, author J. Michael Steele was affiliated with Stanford University. Currently, he is a faculty member at the Statistics Department at the University of Pennsylvania.

This paper is posted at ScholarlyCommons. https://repository.upenn.edu/statistics_papers/25

For more information, please contact repository@pobox.upenn.edu. 


\title{
Hammersley's Law for the Van Der Corput Sequence: An Instance of Probability Theory for Pseudorandom Numbers
}

\author{
Abstract \\ The analogue of Hammersley's theorem on the length of the longest monotonic subsequence of \\ independent, identically, and continuously distributed random variables is obtained for the pseudorandom \\ van der Corput sequence. In this case there is no limit but the precise limits superior and inferior are \\ determined. The constants obtained are closely related to those established in the independent case by \\ Logan and Shepp, and Vershik and Kerov.

\section{Disciplines} \\ Physical Sciences and Mathematics

\section{Comments} \\ At the time of publication, author J. Michael Steele was affiliated with Stanford University. Currently, he is \\ a faculty member at the Statistics Department at the University of Pennsylvania.
}




\title{
HAMMERSLEY'S LAW FOR THE VAN DER CORPUT SEQUENCE: AN INSTANCE OF PROBABILITY THEORY FOR PSEUDORANDOM NUMBERS ${ }^{1}$
}

\author{
By A. Del Junco and J. Michael Steele \\ Ohio State University and Stanford University
}

\begin{abstract}
The analogue of Hammersley's theorem on the length of the longest monotonic subsequence of independent, identically, and continuously distributed random variables is obtained for the pseudorandom van der Corput sequence. In this case there is no limit but the precise limits superior and inferior are determined. The constants obtained are closely related to those established in the independent case by Logan and Shepp, and Vershik and Kerov.
\end{abstract}

1. Introduction. Pseudorandom numbers form the backbone of computer simulation and Monte Carlo analysis, yet there are essentially no known theorems which make explicit the sense in which pseudorandom numbers are replacements for random numbers. There is probably no general result which can be proved, but there is still a program by which a deeper understanding of the relationship between pseudorandom numbers and random numbers can evolve.

For every pseudorandom sequence and every probabalistic theorem, there is surely some analogue of the probabalistic theorem for that pseudorandom sequence. The qualities of that analogue should then accurately reflect the random qualities of the pseudorandom sequence. By a systematic analysis of these pairs of theorems and sequences, a body of results can be obtained which is capable of resolving, or at least eroding, many of the philosophical and practical questions concerning pseudorandom sequences.

This is no overnight task and there is no obvious place to begin other than with the theorems and sequences which interest one most. We have begun with a theorem due to Hammersley and a sequence due to van der Corput.

To introduce Hammersley's theorem, let $X_{i}, i=1,2, \cdots$, be independent random variables with uniform distribution on $[0,1]$. By $l_{n}=l\left(X_{1}, X_{2}, \cdots, X_{n}\right)$ we denote the cardinality of the largest monotone increasing subsequence of the values $X_{1}(\omega), X_{2}(\omega), \cdots, X_{n}(\omega)$. Hammersley [2] proved that

$$
\lim _{n \rightarrow \infty} n^{-\frac{1}{2}} l_{n}=c
$$

where $c$ is a constant and the convergence is in probability. We are particularly interested in (1.1) because of the considerable effort which has been focused on the determination of $c$. Even before convergence had been proved in (1.1), Baer and Brock [1] had conjectured that $c=2$ on the basis of extensive computation. Hammersley [2] gave bounds on $c$ which were improved by Kingman [4], but the

Received September 8, 1977.

${ }^{1}$ Supported in part by Office of Naval Research, Contract N00014-76-C-0475.

AMS 1970 subject classifications. Primary 60C05; secondary 65C10.

Key words and phrases. Van der Corput sequence, monotonic subsequence. 
deepest results published so far are due to Logan and Shepp [6] who proved $c \geqslant 2$. Vershik and Kerov [7] have recently announced that $c \leqslant 2$ but details of their proof have yet to appear.

In the present context Hammersley's theorem has a natural appeal as a substantive probabalistic result in which there is much current interest. The tempting prospects of obtaining $c$ in the analogue of (1.1) for some pseudorandom sequence provide even more reason to start with Hammersley's theorem.

One natural candidate for the sequence of pseudorandom numbers is the sequence generated by the multiplicative congruential method. These are the most widely used pseudorandom numbers but there are several drawbacks to their analysis.

In the first place the sequences generated by the multiplicative congruential method are periodic. Since $l_{n}$ can be properly studied only for $n$ snall compared to the period the asymptotic analysis thus is complicated not only computationally but conceptually.

Primarily for this reason we have begun with the van der Corput sequence. The sequence is generated in a manner almost as simple as multiplicative congruence, but it has the great benefit of being aperiodic. Moreover the sequence has widespread use in numerical integration [3] and considerable historical interest. (See, for example, Knuth's interesting discussion "What is a random sequence" [5] page 127-157.)

To define the van der Corput sequence we first write, for $n \geqslant 0, n=\sum_{i=0}^{\infty} a_{i} 2^{i}$ where $a_{i}=0$ or 1 . The $n+1$ st element of the sequence is $\varphi_{2}(n)=\sum_{i=0}^{\infty} a_{i} 2^{-i-1}$. One can see that $\varphi_{2}(0)=0, \varphi_{2}(1)=\frac{1}{2}, \varphi_{2}(2)=\frac{1}{4}, \varphi_{2}(3)=\frac{3}{4}$, etc. The nature of $\varphi_{2}(n)$ is more easily seen in binary notation where $\varphi_{2}(n)$ is the "the reflection of $n$ in the decimal point." As the subscript suggests one can define a similar sequence by $\varphi_{p}(n)=\sum_{i=0}^{\infty} a_{i} p^{-i-1}$ where $n=\sum_{i=0}^{\infty} a_{i} p^{1}, 0 \leqslant a_{i}<p$ and $p \geqslant 2$ is an integer.

We now have the theorem:

If $l(n)$ is the cardinality of the largest monotone increasing subsequence of $\left\{\varphi_{p}(0), \varphi_{p}(1), \cdots, \varphi_{p}(n-1)\right\}$ then

$$
\lim \inf _{n \rightarrow \infty} n^{-\frac{1}{2}} l(n)=2^{\frac{1}{2}}, \quad \lim \sup _{n \rightarrow \infty} n^{-\frac{1}{2}} l(n)=\frac{3}{2} \quad \text { for } p=2,
$$

and

$\liminf _{n \rightarrow \infty} n^{-\frac{1}{2}} l(n)=2\left(1-p^{-1}\right)^{\frac{1}{2}}, \quad \lim \sup _{n \rightarrow \infty} n^{-\frac{1}{2}} l(n)=p^{\frac{1}{2}} \quad$ for $p>2$.

This result is as precise an analogue to (1.1) as one could realistically expect. Also, the Logan-Shepp lower bound on $c$ makes it particularly noteworthy that

$$
\lim _{p \rightarrow \infty} \lim . \text { inf }_{n \rightarrow \infty} n^{-\frac{1}{2}} l(n)=2 .
$$

For digestibility, the proof of these results has been divided into four parts. First we obtain a geometrical characterization of the monotone subsequences of van der Corput's sequence. Upper and lower bounds are then obtained for $l(n)$. Finally the required limits are identified. 
2. The geometrical representation. Our first goal is to obtain a representation from which detailed information about $\left\{\varphi_{p}(0), \varphi_{p}(1), \cdots, \varphi_{p}(n-1)\right\}$ can be deduced. We define $\sigma_{n}$ to be the unique permutation of $\{0,1, \cdots, n-1\}$ which puts $\varphi_{p}(i), 0 \leqslant i \leqslant n-1$ in increasing order, i.e.,

$$
\varphi_{p}\left(\sigma_{n}(0)\right)<\varphi_{p}\left(\sigma_{n}(1)\right)<\cdots<\varphi_{p}\left(\sigma_{n}(n-1)\right) .
$$

This permutation will be written as a sequence

$$
\sigma_{n}=\left(\sigma_{n}(0), \sigma_{n}(1), \cdots, \sigma_{n}(n-1)\right)
$$

and our reason for introducing $\sigma_{n}$ is the elementary fact that the length of the longest monotone increasing subsequence of $\sigma_{n}$ is equal to $l(n)$.

We also have the following law of formation of $\sigma_{m p^{n}}$ which will be crucial.

LEMMA 2.1. One obtains $\sigma_{m p^{n}}$ from $\sigma_{p^{n}}$ by replacing the entry $\sigma_{p^{n}}(i)$ of $\sigma_{p^{n}}$ by the sequence $\sigma_{p^{n}}(i)+p^{n} \sigma_{m}$.

REMARK. Here and in the following if $x$ is a sequence then $a+b x$ is a sequence with the same domain as $x$ defined by $(a+b x)(i)=a+b x(i)$.

Proof OF LEMMA. Notice that there are two things we must show:

$$
\varphi_{p}\left(\sigma_{p^{n}}(i)+p^{n} \sigma_{m}(j)\right)<\varphi_{p}\left(\sigma_{p^{n}}(i)+p^{n} \sigma_{m}(j+1)\right),
$$

and

$$
\varphi_{p}\left(\sigma_{p^{n}}(i)+p^{n} \sigma_{m}(j)\right)<\varphi_{p}\left(\sigma_{p^{n}}(i+1)+p^{n} \sigma_{m}\left(j^{\prime}\right)\right)
$$

for any $j, j^{\prime}$.

We first suppose $j<p^{n}$ and $k$ is any integer. Setting $j=\sum_{i=0}^{n-1} a_{i} p^{i}, k=$ $\sum_{i=n}^{m} a_{k} p^{i-n}$ we have $j+p^{n} k=\sum_{i=0}^{m} a_{i} p^{1}$, and consequently

$$
\begin{aligned}
\varphi_{p}\left(j+p^{n} k\right) & =\sum_{i=0}^{m} a_{i} p^{-(i+1)} \\
& =\sum_{i=0}^{n-1} a_{i} p^{-(i+1)}+p^{-n} \sum_{i=n}^{m} a_{i} p^{-(i+1)} \\
& =\varphi_{p}(j)+p^{-n} \varphi_{p}(k) \text { for } j<p^{n}
\end{aligned}
$$

Now by (2.3) we see (2.1) is equivalent to

$$
p^{-n} \varphi_{p}\left(\sigma_{m}(j)\right)<p^{-n} \varphi_{p}\left(\sigma_{m}(j+1)\right)
$$

which is just the definition of $\sigma_{m}$. Similarly, (2.2) is equivalent to

$$
\varphi_{p}\left(\sigma_{p^{n}}(i)\right)+p^{-n} \varphi_{p}\left(\sigma_{m}(j)\right)<\varphi_{p}\left(\sigma_{p^{n}}(i+1)\right)+p^{-n} \varphi_{p}\left(\sigma_{m}\left(j^{\prime}\right)\right) .
$$

To check (2.4) we note that, as $i$ runs through $\left\{0,1, \cdots, p^{n}-1\right\}, \varphi_{p}(i)$ runs through $\left\{0, p^{-n}, 2 p^{-n}, \cdots,\left(p^{n}-1\right) p^{-n}\right\}$ so that, in fact, $\varphi_{p}\left(\sigma_{p^{n}}(i+1)\right)=p^{-n}+$ $\varphi_{p}\left(\sigma_{p^{n}}(i)\right)$. The proof of the lemma is thus complete.

In many ways it is easier to work with the permutation matrix $A_{n}$ associated with $\sigma_{n}$. We recall that $A_{n}$ is an $n \times n$ matrix defined by

$$
\begin{aligned}
A_{n}(i, j) & =1 & & \text { if } i=\sigma_{n}(j) \\
& =0 & & \text { otherwise. }
\end{aligned}
$$


To rephrase Lemma 2.1 in terms of matrices we define $m$ different $p^{n} \times m p^{n}$ matrices $\bar{A}_{p^{n}}^{0}, \bar{A}_{p^{n}}^{1}, \cdots, \bar{A}_{p^{n}}^{m-1}$ by

$$
\begin{aligned}
{\overline{A_{p^{n}}}}^{0}(i, j) & =A_{p^{n}}\left(i, j m^{-1}\right) \text { if } m \mid j \\
& =1 \quad \text { otherwise }
\end{aligned}
$$

and

$$
\bar{A}_{p^{n}}^{l}(i, j)={\overline{A_{p}}}^{0}(i,(j-l) \bmod m)
$$

Intuitively, we get $\overline{A_{p^{n}}^{0}}$ by inserting $m-1$ columns of 0 's after each column of $A_{p^{n}}$ and then shift $\bar{A}_{p^{n}}^{0}$ by $l$ places to the right to get $\bar{A}_{p^{n}}^{l}$.

Finally we can phrase Lemma 2.1 by the following formula for $A_{m p^{n}}$ as a block matrix:

$$
A_{m p^{n}}=\left[\begin{array}{c}
\bar{A}_{p^{n}}^{\sigma_{m}^{-1}(1)} \\
\bar{A}_{p^{n}}^{\sigma_{m}^{-1}(2)} \\
\vdots \\
\bar{A}_{p^{n}}^{\sigma_{m}^{-1}(m)}
\end{array}\right]
$$

This matrix representation of the permutation permits a geometrical view of the decreasing subsequences. Formally an increasing subsequence of $\sigma_{n}$ is a sequence of integers $P=\left\{j_{1}<j 2<\cdots<j_{l}\right\}$ such that $\sigma\left(j_{i}\right)<\sigma\left(j_{i+1}\right)$. Such a subsequence can be identified in $A_{n}$ as a path of 1's which goes down and to the right. The set of integers $\left[j_{1}, j_{l}\right]=\left\{k: j_{1} \leqslant k \leqslant j_{l}\right\}$ will be called the domain of the path $P$ and will be denoted by $\operatorname{dom}(P)$. The integer interval $\left[\sigma_{n}\left(j_{1}\right), \sigma_{n}\left(j_{l}\right)\right]=\left\{k: \sigma_{n}\left(j_{1}\right)\right.$ $\left.\leqslant k \leqslant \sigma_{n}\left(j_{l}\right)\right\}$ will be called the range of the path, and naturally $l(P)$ will be called the length of $P$.

3. The lower bound lemma. The main result of this section is the following:

LEMMA 3.1. For $p^{n-2} \leqslant m \leqslant p^{n-1}$ we have

$$
l\left(m p^{n}\right) \geqslant p^{n-1}+m(p-1)-(3 p-1),
$$

and for $p^{n-3} \leqslant m \leqslant p^{n-2}$ we have

$$
l\left(m p^{n}\right) \geqslant p^{n-3}(p-1)+m p-\left(p^{2}+2 p-1\right) .
$$

Proof. First we will consider $m$ such that $p^{n-2} \leqslant m \leqslant p^{n-1}$ and construct an explicit path through $A_{m p^{n}}$. By setting $m_{1}=\left(p^{n-1}-m\right) /(p-1)$ and $m_{2}=m-$ $m_{1}$ we have $m_{1} p^{2}+m_{2} p=p^{n}$. For $0 \leqslant i<\left[m_{1}\right]$ we can define a path $P_{i}$ through $A_{p^{n}}$ of length $2 p-1$ and domain $\left[p^{2} i, p^{2}(i+1)-1\right]$ by

$$
\begin{gathered}
P_{i}=\left(p^{2} i, p^{2} i+p, p^{2} i+2 p, \cdots, p^{2}(i+1)-p,\right. \\
\left.p^{2}(i+1)-p+1, \cdots, p^{2}(i+1)-1\right) .
\end{gathered}
$$

For $0 \leqslant i<\left[m_{2}\right]$ we then define a path $P_{\left[m_{1}\right]+i}$ through $A_{p^{n}}$ with length $p$ and domain $\left[\left[m_{1}\right] p^{2}+i p,\left[m_{1}\right] p^{2}+(i+1) p-1\right]$ by

$$
P_{\left[m_{1}\right]+i}=\left(\left[m_{1}\right] p^{2}+i p,\left[m_{1}\right] p^{2}+i p+1, \cdots,\left[m_{1}\right] p^{2}+(i+1) p-1\right) .
$$


Now we consider the paths $\bar{P}_{i}$ in $\bar{A}_{p^{m}}^{\sigma_{m}^{-1}(i)}$ which correspond to $P_{i}$. These paths are defined by $\bar{P}_{i}=m P_{i}+\sigma_{m}^{-1}(i)$. Finally we define our desired path $P$ through $A_{m p^{n}}$ as the concatenation of the $\bar{P}_{i}$,

$$
P=\bar{P}_{0} \bar{P}_{1} \cdots \bar{P}_{\left[m_{1}\right]+\left[m_{2}\right]-1} .
$$

To make certain that the path $P$ is properly defined we have to check that for each $k \in \operatorname{dom} \bar{P}_{i+1}$ and $l \in \operatorname{dom} \bar{P}_{i}$ we have $k>l$. This condition will be abbreviated by dom $\bar{P}_{i+1}>\operatorname{dom} \bar{P}_{i}$, and it follows immediately from the corresponding fact that $\operatorname{dom} P_{i+1}>\operatorname{dom} P_{i}$. By our construction we finally have

$$
\begin{aligned}
l\left(m p^{n}\right) & \geqslant l(P)=\left[m_{1}\right](2 p-1)+\left[m_{2}\right] p \\
& \geqslant m_{1}(2 p-1)+m_{2} p-(3 p-1) \\
& =p^{n-1}+m(p-1)-(3 p-1)
\end{aligned}
$$

which completes the proof of (3.1).

We now consider $m, p^{n-3} \leqslant m \leqslant p^{n-2}$ and begin by setting $m_{1}=\left(p^{n-2}-\right.$ $m) /(p-1)$ and $m_{2}=m-m_{1}$. This time we have $m_{1} p^{3}+m_{2} p^{2}=p^{n}$. As in our previous construction there is a path of length $p^{2}$ through $A_{p^{3}}$, so for $0 \leqslant i<\left[m_{1}\right]$ there is a path $P_{i}$ of length $p^{2}$ through $A_{p^{n}}$ with domain $\left[i p^{3},(i+1) p^{3}-1\right]$. For $0 \leqslant i<\left[m_{2}\right]$ there is a path $P_{\left[m_{1}\right]+i}$ of length $2 p-1$ through $A_{p^{n}}$ with domain $\left[\left[m_{1}\right] p^{3}+i p^{2},\left[m_{1}\right] p^{3}+(i+1) p^{2}-1\right]$. Next we set $\bar{P}_{i}=m P_{i}+\sigma_{m}^{-1}(i)$ and obtain a path through ${\overline{A_{p}^{m}}}_{\sigma^{-1}(i)}^{\sigma^{2}}$ by letting

$$
P=\bar{P}_{0} \bar{P}_{1} \cdots \bar{P}_{\left[m_{1}\right]+\left[m_{2}\right]-1 .}
$$

Finally we calculate as before:

$$
\begin{aligned}
l\left(m p^{n}\right) & \geqslant l(P)=\left[m_{1}\right] p^{2}+\left[m_{2}\right](2 p-1) \\
& \geqslant m_{1} p^{2}+m_{2}(2 p-1)-\left(p^{2}+2 p-1\right) \\
& =p^{n-2}(p-1)+m p-\left(p^{2}+2 p-1\right)
\end{aligned}
$$

which completes the proof of (3.2).

REMARK. The preceding proof probably appears more involved than an examination of an example would indicate. By calculating $A_{m p^{n}}$ for $p=3$ and several small values of $m$ and $n$ one can easily find the path $P$ and see how it evolves as $m$ and $n$ increase. Similarly in the arguments which follow one should keep the example $p=3$ clearly in mind, perhaps by keeping a small table of the $A_{m p^{n}}$.

4. The upper bound lemma. The recursive nature of $A_{m p^{n}}$ provides the key to the following, Lemma 4.1. For any positive integer $m$ we have

$$
l\left(m p^{n-1}\right) \leqslant p^{n-1}+m(p-1)
$$

and

$$
l\left(m p^{n}\right) \leqslant p^{n-1}-p^{n-2}+m p .
$$


Proof. For any path $P$ through $A_{m p^{n}}$ we can decompose $P$ as a concatenation

$$
P=\bar{P}_{0} \bar{P}_{1} \cdots \bar{P}_{m-1}
$$

where $\bar{P}_{i}$ is a path through $\bar{A}_{p^{m^{\prime}}}^{\sigma^{-1}(i)}$. Here one should note that the range of $\bar{P}_{i}$ is a subset of $\left[i^{n},(i+1) p^{n}-1\right]$.

We now let $P_{i}$ be the corresponding path in $A_{p^{n}}$ of $\bar{P}_{i}$. That is, we let $P_{i}=m^{-1}\left(\bar{P}_{i}\right.$ $\left.-\sigma_{m}^{-1}(i)\right)$. We do not have $\operatorname{dom} P_{i}<\operatorname{dom} P_{i+1}$ but we obviously do have $\operatorname{dom} P_{i}$ $\leqslant \operatorname{dom} P_{i+1}$ so the two paths have at most one common point. If $\operatorname{dom} P_{i} \cap$ $\operatorname{dom} P_{i+1} \neq \varnothing$ we shall say $P_{i}$ and $P_{i+1}$ are linked. Since $l(P) \leqslant \sum_{i=0}^{m-1} l\left(P_{i}\right)$ the lemma would follow from $\sum_{i=0}^{m-1} l\left(P_{i}\right) \leqslant \min \left(p^{n-1}+m(p-1), p^{n-1}-p^{n-2}+\right.$ $m p)$. This fact will be proved using only the inequality dom $P_{i} \leqslant \operatorname{dom} P_{i+1}$.

First we decompose $A_{p^{n}}$ into $p^{n}$ blocks of height $p^{n-1}$ and width $p$. We note that each block has exactly one 1, and the pattern of 1's in each column of blocks is the same as the pattern in $A_{p}$.

The block width $w(P)$ of a path $P$ is defined as the number of distinct columns of blocks which intersect $P$. The block height $h(P)$ is defined correspondingly. Further, we let $\mu$ equal the number of integers $i$ such that $P_{i}$ is linked to $P_{i+1}$.

If $P_{i}$ and $P_{i+1}$ are linked we define their linkage as the concatenation $P_{i} P_{i+1}^{*}$ where $P_{i+1}^{*}$ denotes $P_{i}$ with its first entry removed. By forming the successive linkage of the $P_{i}$ we obtain $m-\mu$ paths $\hat{P}_{1}, \hat{P}_{2}, \cdots, \hat{P}_{m-\mu}$ which are unlinked and for which

$$
\mu+\sum_{i=1}^{m-\mu} l\left(\hat{P}_{i}\right)=\sum_{i=0}^{m-1} l\left(P_{i}\right)
$$

Next we form all possible concatenations of successive $\hat{P}_{i}$ to get $s$ paths $P_{1}^{\prime}, P_{2}^{\prime}, \cdots, P_{s}^{\prime}$ which allow no further concatenation. One then has $s \leqslant m-\mu$ and

$$
\sum_{i=1}^{s} l\left(P_{i}^{\prime}\right)=\sum_{i=1}^{m-\mu} l\left(\hat{P}_{i}\right) .
$$

Moreover, one notes that no two $P_{i}^{\prime}$ share a common column of blocks for the simple reason that $\operatorname{dom} P_{i}<\operatorname{dom} P_{i+1}$ and the 1 in any block is to the right of the 1 in any block above it.

The crucial observation is that for each path $P_{i}^{\prime}$ we have

$$
l\left(P_{i}^{\prime}\right) \leqslant h\left(P_{i}^{\prime}\right)+w\left(P_{i}^{\prime}\right)-1 \leqslant p+w\left(P_{i}^{\prime}\right)-1
$$

which by summing over $i$ yields

$$
\sum_{i=1}^{s} l\left(P_{i}^{\prime}\right) \leqslant \sum_{i=1}^{s} w\left(P_{i}^{\prime}\right)+s(p-1) .
$$

Since no two $P_{i}^{\prime}$ share a column of blocks we have

$$
\sum_{i=1}^{s} w\left(P_{i}^{\prime}\right) \leqslant p^{n-1} .
$$

By the inequality (4.5) we obtain

$$
\sum_{i=1}^{s} l\left(P_{i}^{\prime}\right) \leqslant p^{n-1}+s(p-1)
$$

which by setting $s=m-\mu-\lambda$ with $\lambda \geqslant 0$ using (4.3) and (4.4) becomes

$$
\sum_{i=0}^{m-1} l\left(P_{i}\right) \leqslant p^{n-1}+(m-\lambda)(p-1)-\mu(p-2) .
$$


This completes the proof of (4.1) since $\lambda \geqslant 0$ and $\mu \geqslant 0$. On the other hand (4.6) also yields $s \leqslant p^{n-1}$ so $-\mu \leqslant p^{n-1}-(m-\lambda)$ which by (4.8) implies

$$
\begin{aligned}
\sum_{i=0}^{m-1} l\left(P_{i}\right) & \leqslant p^{n-1}+(m-\lambda)(p-1)+\left(p^{n-1}-(m-\lambda)\right)(p-2) \\
& \leqslant p^{n}-p^{n-1}+m .
\end{aligned}
$$

This implies $l\left(m p^{n}\right) \leqslant p^{n}-p^{n-1}+m$ and by replacing $n$ by $n-1$ and $m$ by $m p$ we obtain the proof of (4.2). This completes our proof of the upper bound lemma.

By applying the preceding lemmas, we should note that in certain cases the inequalities can be made to provide equality. By inspecting the proof of (3.1) in the cases of $m=p^{n-1}$ and $m=p^{n-2}$ one sees that it is not necessary to estimate greatest integers so that the $3 p-1$ disappears from the right of (3.1). Combining this with (4.1) we have the identities

$$
l\left(p^{2 n-1}\right)=p^{n} \text { and } l\left(p^{2 n-2}\right)=p^{n-2}(2 p-1) .
$$

By similar analysis for $p=2$ we also have the identity

$$
\begin{array}{rlrl}
l\left(m 2^{n}\right) & =2^{n-1}+m & & \text { for } \quad 2^{n-2} \leqslant m \leqslant 2^{n-1} \\
& =2^{n-1}+2 m & \text { for } \quad 2^{n-3} \leqslant m \leqslant 2^{n-2} .
\end{array}
$$

5. Identification of the limits. To complete the proof of our theorem, we first identify $\lim \sup _{n \rightarrow \infty} N^{-\frac{1}{2} l} l(N)$ where $N$ is restricted to $S=\left\{m p^{n}: p^{n-3} \leqslant m \leqslant\right.$ $\left.p^{n-1}\right\}$.

LEMMA 5.1. Setting $K=\max \left(2\left(1-p^{-1}\right)^{\frac{1}{2}}, p^{\frac{1}{2}}, 2-p^{-1}\right)$ and $k=\min (2(1-$ $\left.\left.p^{-1}\right)^{\frac{1}{2}}, p^{\frac{1}{2}}, 2-p^{-1}\right)$, we have

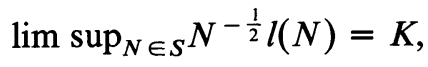

and

$$
\lim \inf _{N \in S} N^{-\frac{1}{2}} l(N)=k .
$$

Proof. As in Section 3 we deal with $p^{n-2} \leqslant m \leqslant p^{n-1}$ (case 1) and $p^{n-3} \leqslant m$ $\leqslant p^{n-2}$ (case 2) separately. This time we begin with case 2. By (3.2) and (4.2) we have

$$
g_{n}(m)-\left(p^{2}+2 p+1\right)\left(m p^{n}\right)^{-\frac{1}{2}} \leqslant l\left(m p^{n}\right)\left(m p^{n}\right)^{-\frac{1}{2}} \leqslant g_{n}(m)
$$

where $g_{n}(x)=\left(p^{n-2}(p-1)+x p\right)\left(x p^{n}\right)^{-\frac{1}{2}}$. Consequently we have

$$
\lim \inf l\left(m p_{n}\right)\left(m p^{n}\right)^{-\frac{1}{2}}=\lim \inf g_{n}(m)
$$

where the lim inf is taken over the set $S_{1}=\left\{m p^{n}: p^{n-3} \leqslant m \leqslant p^{n-2}, 1 \leqslant n\right\}$. One has the same equation as (5.3) for. the lim sup.

Next observe $g_{n}(m)$ has its minimum at $a_{n}=p^{n-3}(p-1)$ and $g_{n}$ is decreasing for $p^{n-3} \leqslant x \leqslant a_{n}$ and increasing for $a_{n} \leqslant x \leqslant p^{n-2}$. One easily checks that $g_{n}\left(p^{n-3}\right)=p^{\frac{1}{2}}, g_{n}\left(p^{n-2}\right)=2-p^{-1}$, and $g_{n}\left(a_{n}\right)=2\left(1-p^{-1}\right)^{\frac{1}{2}}$ so we have

$$
\lim \inf _{m p^{n} \in S_{1}} g_{n}(m)=k \text { and } \lim \sup _{m p^{n} \in S_{1}} g_{n}(m)=K \text {. }
$$


To prove the comparable identities for case 1 one sets $f_{n}(x)=\left(p^{n-1}+x(p-\right.$ 1)) $\left(x p^{n}\right)^{-\frac{1}{2}}$ and obtains from (3.1) and (4.1) that

$$
\lim \inf _{m p^{n} \in S_{2}} l\left(m p^{n}\right)\left(m p^{n}\right)^{-\frac{1}{2}}=\lim \sup _{m p^{n} \in S_{2}} f_{n}(m)
$$

where $S_{2}=\left\{m p^{n}: p^{n-2} \leqslant m p^{n-1}, n \geqslant 1\right\}$. The same equality holds for the limsup, and we note as before that $f_{n}$ has a minimum at $p^{n-1}(p-1)^{-1}=b_{n}$, decreases for $p^{n-2} \leqslant x \leqslant b_{n}$ and increases for $b_{n} \leqslant x \leqslant p^{n-1}$. Finally we note $f_{n}\left(b_{n}\right)=2(1-$ $\left.p^{-1}\right)^{\frac{1}{2}}, f_{n}\left(p^{n-1}\right)=p^{\frac{1}{2}}$, and $f_{n}\left(p^{n-2}\right)=2-p^{-1}$. Hence, we have

$$
\lim \inf _{m p^{n} \in S_{2}} f_{n}(m) \geqslant k \text { and } \lim \sup _{m p^{n} \in S_{2}} f_{n}(m) \leqslant K \text {. }
$$

One has inequality in (5.5) since $b_{n}$ is not an integer. Actually equality can be proved but is not required for the rest of the proof. By (5.3), (5.4) and (5.5) we have completed the proof of (5.1) and (5.2) as required by the lemma.

The proof of our theorem can now be completed in a routine way. For an arbitrary integer $N$ we write

$$
N=m p^{n}+r \text { with } p^{n-3} \leqslant m \leqslant p^{n-1} \text { and } 0 \leqslant r \leqslant p^{n+2} .
$$

Letting $l(i, j)$ denote the cardinality of the largest increasing subsequence of $\left(\varphi_{p}(i), \varphi_{p}(i+1), \cdots, \varphi_{p}(j-1)\right)$ we see

$$
l\left(i p^{k},(i+1) p^{k}\right)=l\left(p^{k}\right)
$$

as a consequence of (2.3). There is also the obvious fact that $l(i, j)$ is subadditive,

$$
l(i, k) \leqslant l(i, j)+l(j, k) \quad \text { for } i \leqslant j \leqslant k .
$$

By choosing an integer $t$ such that $t p^{n+2} \leqslant m p^{n}$ and $m p^{n}+p^{n+2} \leqslant(t+2) p^{n+2}$ one has by (5.6) that

$$
\begin{aligned}
l(N) & \leqslant l\left(m p^{n}+p^{n+2}\right) \\
& \leqslant l\left(m p^{n}\right)+l\left(m p^{n}, m p^{n}+p^{n+2}\right) \\
& \leqslant l\left(m p^{n}\right)+l\left(t p^{n+2},(t+2) p^{n+2}\right) \\
& \leqslant l\left(m p^{n}\right)+2 l\left(p^{n+2}\right) .
\end{aligned}
$$

Hence we have

$$
l\left(m p^{n}\right) \leqslant l(N) \leqslant l\left(m p^{n}\right)+2 l\left(p^{n+2}\right) .
$$

Since for large $N$ one has $N / m p^{n}$ is near 1 and $l\left(p^{n+2}\right) /\left(m p^{n}\right)^{\frac{1}{2}}$ is near 0 , the inequality (5.8) together with Lemma 5 completes the proof of the theorem.

\section{REFERENCES}

[1] BAer, R. M. and Brock, P. (1968). Natural sorting over permutation spaces. Math. Comp. 22 385-410.

[2] Hammersley, J. M. (1972). A few seedlings of research. Proc. Sixth Berkeley Symp. Math. Statist. Probability 1 345-394. Univ. of California Press.

[3] Hammersley, J. M. and Handscomb, D. C. (1964). Monte Carlo Methods. Methuen, London. 
[4] Kingman, J. F. C. (1973). Subadditive ergodic theory. Ann. Probability 1 883-899.

[5] KNuth, D. E. (1969). The Art of Computer Programming, 2 (Seminumerical Algorithms). AddisonWeslay, Palo Alto.

[6] LogAN, B. F. and SHEPP, L. A. (1975). A variational problem for random Young tableaux. Advances in Math. 26 206-222.

[7] Vershik, A. M. and Kerov, S. V. (1977). Asymptotics of the Plancherel measure of the symmetric group and the limiting form of Young tables. Soviet Math. Dokl. 18 527-531.

DePARTMENT OF MATHEMATICS

OHIO STATE UNIVERSITY

Columbus, OHo 43210
DePaRTMENT OF Statistics

STANFORD UNIVERSITY

StaNFord, CaLIFornia 94305 